\title{
Comparison of transesophageal and intracardiac echocardiography in guiding percutaneous left atrial appendage closure with an Amplatzer Amulet device
}

\author{
Witold Streb, Katarzyna Mitręga, Tomasz Podolecki, Stanisław Morawski, Mariola Szulik, \\ Ewa Jędrzejczyk-Patej, Tomasz Kukulski, Zbigniew Kalarus \\ $1^{\text {st }}$ Department of Cardiology, Congenital Heart Diseases and Electrotherapy, Silesian Center for Heart Diseases in Zabrze, \\ Medical University of Silesia in Katowice, Poland
}

Adv Interv Cardiol 2019; 15, 4 (58): 446-454 DOI https://doi.org/10.5114/aic.2019.90219

\begin{abstract}
Introduction: Percutaneous occlusion of the left atrial appendage (LAAO) is becoming an extensively used method of stroke prevention in individuals with contraindications to oral anticoagulants. Transesophageal echocardiography (TOE) is the gold standard for LAAO guiding, but intracardiac echocardiography (ICE) appears to be a potential alternative.

Aim: To compare the LAAO procedure guided by TOE or ICE with respect to procedural success and safety.

Material and methods: TOE-guided LAAO was performed in 12 patients and ICE-guided LAAO in 11 patients. ICE was performed using an 8F AcuNav probe and the ACUSON SC2000 system. For LAAO the Amplatzer Amulet was used. After 1 month TOE was performed.

Results: Procedural success was achieved in all patients in TOE and ICE groups. There was 1 complication (groin hematoma). The procedure time was significantly longer in the TOE group (43 to $80 \mathrm{~min}$; median: $54 \mathrm{~min}$ ) compared to the ICE group (28 to 67 min; median: $45 \mathrm{~min}),(p=0.02)$ The time needed to puncture the interatrial septum and time needed to remove the sheath did not differ between groups. Fluoroscopic time was insignificantly longer in the ICE group (9.91 $\pm 4.01 \mathrm{~s})$ compared to the TOE group (7.69 $\pm 3.21 \mathrm{~s})$, and a significantly larger contrast media volume was used in the ICE group (30.00 $\pm 6.67 \mathrm{ml} \mathrm{vs.} 40.45 \pm 23.18 \mathrm{ml}, p=0.03)$. There were no statistically significant differences in the results between TOE and ICE groups in follow-up assessments.

Conclusions: LAAO using the Amplatzer Amulet may be successfully and safely guided by ICE. ICE offered shorter procedure time and similar results irrespectively of left atrial appendage anatomy compared to TOE guidance.
\end{abstract}

Key words: transesophageal echocardiography, left atrial appendage occlusion, intracardiac echocardiography.

Su m m a ry

The current study aimed to compare the course and results of the left atrial appendage (LAAO) procedure guided by transesophageal echocardiography (TOE) or intracardiac echocardiography (ICE) particularly with respect to procedural success and safety, but also with regard to the optimal effect of the implantation. We found that the procedure time was significantly longer in the TOE group. However, the fluoroscopic time and contrast media used were insignificantly longer in the ICE group compared to the TOE group. The optimal implantation effect was obtained regardless of whether TOE or ICE was used.

\section{Introduction}

During the last years percutaneous occlusion of the left atrial appendage (LAAO) has become an extensively used method of stroke prevention in individuals with contraindications to oral anticoagulants. The proper vi- sualization of the left atrium is crucial for both procedure planning and guiding. However, imaging of the left atrial appendage remains challenging with respect to assessment of left atrial anatomy as well as for guiding of the LAAO procedure. In the preliminary assessment of the left atrium the most commonly used imaging modality

\section{Corresponding author:}

Witold Streb MD, $1^{\text {st }}$ Department of Cardiology, Congenital Heart Diseases and Electrotherapy, Silesian Center for Heart Diseases, Medical University of Silesia, 2 Szpitalna St, 41-800 Zabrze, Poland, phone: +48 531000 414, e-mail: streb@wp.pl

Received: 3.02.2019, accepted: 8.07.2019. 
is transesophageal echocardiography (TOE), particularly when using 3-dimensional echocardiography [1]. Also TOE remains the gold standard for exclusion of thromboembolic material within the left atrium and in its appendage [2, 3]. Nevertheless, some authors emphasize the advantages of computed tomography or cardiac magnetic resonance for exploration of the anatomy of the left atrial appendage and for the precise measurement of its dimensions [4, 5]. Thus the debate on the optimal imaging strategy for the pre-procedural visualization of the left atrium is still open.

Less attention was paid for the comparison of imaging techniques which are potentially useful in monitoring the LAAO procedures. The fundamental one is fluoroscopy. However, it has many disadvantages. First of all it allows one to obtain only mono-plane images. Secondly, soft tissue is not visible, hence the visualization of the left atrial appendage requires an injection of contrast agents. Therefore most centers reach for support from transesophageal echocardiography. This semi-invasive technique overcomes the limitations of fluoroscopy and is safe. It delivers valuable data regarding the dimensions of the left atrial appendage and allows the post-deployment assessment including the interference of the occluder with adjacent structures or the registration of residual flow within the lobe of the appendage. Based on fluoroscopy and transesophageal echocardiography some newer techniques have been developed, such as fusion imaging, facilitating further the navigation of the LAAO as well as improving the results of occluder implantation [6].

Nevertheless, the navigation of the LAAO with transesophageal echocardiography requires anesthesia or sedation of the patient. Taking into account that patients referred for LAAO are suffering from multiple disorders, both sedation and anesthesia raise the higher risk of complications for them compared to the general population. Among them there are also patients with absolute contraindications to TOE (e.g., esophagectomy) or those with relative contraindications, e.g. hepatic illnesses and esophageal varices.

Intracardiac echocardiography (ICE) similar to TOE may be potentially useful for the navigation of the LAAO procedure. However, evidence that ICE guidance can be equally effective is still lacking.

\section{Aim}

The current study aimed to compare the course of the LAAO procedure guided by TOE or ICE particularly with respect to procedural success and safety.

\section{Material and methods}

Among patients diagnosed with atrial fibrillation and referred for LAAO, the echocardiographic eligibility criteria for LAAO were assessed by TOE. After this preliminary selection, 23 patients agreed to participate in the study. All of these patients had both an increased risk of stroke (CHADSVASc score $\geq 2$ ), and contraindications for oral anticoagulation. Informed consent was obtained from each individual for performing the procedure of percutaneous left atrial appendage occlusion as a method of stroke prophylaxis. The patients were informed about the advantages of LAAO and potential risk of the procedure. It was also explained that LAAO would be guided by either transesophageal echocardiography or intracardiac echocardiography. The imaging modality used for guidance of LAAO was chosen based on the order of patients enrolled in the study. Thus each second patient enrolled in the study had LAAO guided by ICE, and the remaining ones by TOE.

\section{Procedure}

For LAAO the Amplatzer Amulet occluder was used. The size of occluder chosen for LAAO was based on the results of measurements performed during the TOE prior to the procedure and compared with the fluoroscopic measurements made during LAAO. The occluder used was 2-4 $\mathrm{mm}$ larger than the landing zone diameter according to the suggestions in the occluder sizing charts distributed by the producer.

The vascular access was obtained by the puncture of the right femoral vein. Later the Brockenbrough needle passed through the interatrial septum in its lower, posterior portion. The site of puncture was guided either by TOE or ICE. Later the sheath was changed for that dedicated for the delivery of the Amplatzer Amulet - $12 \mathrm{~F}$ or $14 \mathrm{~F}$ sheath - depending on the occluder size.

The device was positioned in the neck of the left atrium appendage under echocardiographic (TOE or ICE) and fluoroscopic guidance. The criteria of proper implantation included:

$-2 / 3$ of the occluder lobe placed below the landing zone, - the axis of the occluder parallel to the axis of the left atrial appendage neck,

- compression of the lobe of the occlude,

- lack of protrusion of the disk above the orifice of the left atrial appendage,

- separation between the lobe and disk of the device,

- lack or a trace of leak in the left atrial appendage after implantation.

After removal of the catheter from the vein, the hemostatic suture was applied, followed by the compression bandage left for $3 \mathrm{~h}$.

\section{Transesophageal echocardiography}

The electrocardiographic (ECG)-gated TOE was performed using Vivid E9 (6 VT-D probe; $5 \mathrm{MHz}$ ). 3-dimensional TOE zoom images were obtained from one ECG cycle in two-chamber view. Then gain was turned to optimize the images with particular care not to allow 
for a drop-out effect. The landing zone area was reconstructed by the alignment of three perpendicular planes at the level of the circumflex artery using the multi-plane technique. The measurements were taken during the left atrial appendage filling period. The largest diameter of the left atrial appendage landing zone was used as a reference for the selection of occluder size. During the procedure the X-plane views were used for the navigation of the transseptal puncture and for monitoring of device deployment in the left appendage neck. After that the sealing effect was confirmed by the lack of flow within the appendage by color using Doppler with the scale reduced $<30 \mathrm{~cm} / \mathrm{s}$.

\section{Intracardiac echocardiography}

ICE imaging was performed using a single-use, $8 \mathrm{~F}$ AcuNav Diagnostic Ultrasound Catheter (Siemens Medical Solutions, USA) and ACUSON SC2000 ultrasound system (Siemens Medical Solutions USA, Inc.). An AcuNav catheter was inserted by the puncture of the left femoral vein. The ICE probe was linked to the ultrasound system by a reusable SwiftLink connector. The catheter handle and the SwiftLink were placed in a sterile cover. The AcuNav handle allows one to change the position of a tip of a catheter in four directions (anterior, posterior, left and right). It was introduced to the heart chambers via the puncture of the left femoral vein.

With the tip of the ICE probe placed in the right atrium the probe was turned clock-wise and banded posterior to obtain a view of the septum. After the trans- septal puncture the sheath for transseptal puncture was exchanged with the sheath and dilator designed for the introduction of Amplatzer Amulet. With a wire placed in the left upper pulmonary vein the Amplatzer sheath was placed in the left atrium and then withdrawn. Crossing the interatrial septum with a sheath and its dilator was repeated 2-3 times. This maneuver facilitated the passage of the ICE probe via the interatrial septum. Crossing of the ICE probe through the interatrial septum required the adjustment of its position in the right atrium by manipulation of its handle and alignment with the wire put in the left upper pulmonary vein under fluoroscopy in a different view. When advanced in the left atrium the ICE probe was maneuvered to achieve the best left atrial appendage view (Figure 1). Typically it was obtained with an ultrasound catheter deflected in the posterior direction and with clockwise rotation. Following the expansion of the occluder, its position was assessed and lack of flow within the appendage was confirmed by using color Doppler with the scale reduced $<30 \mathrm{~cm} / \mathrm{s}$.

\section{Patient preparation}

Prior to the procedure all patients received a loading dose of aspirin and clopidogrel if not already on treatment. A prophylactic cefazolin dose and $0.9 \%$ saline infusion was given to all subjects prior to LAAO. General anesthesia was used in the group of patients who had LAAO under the guidance with TOE. In patients in whom the procedure was guided by ICE only local anesthesia without sedation was used.
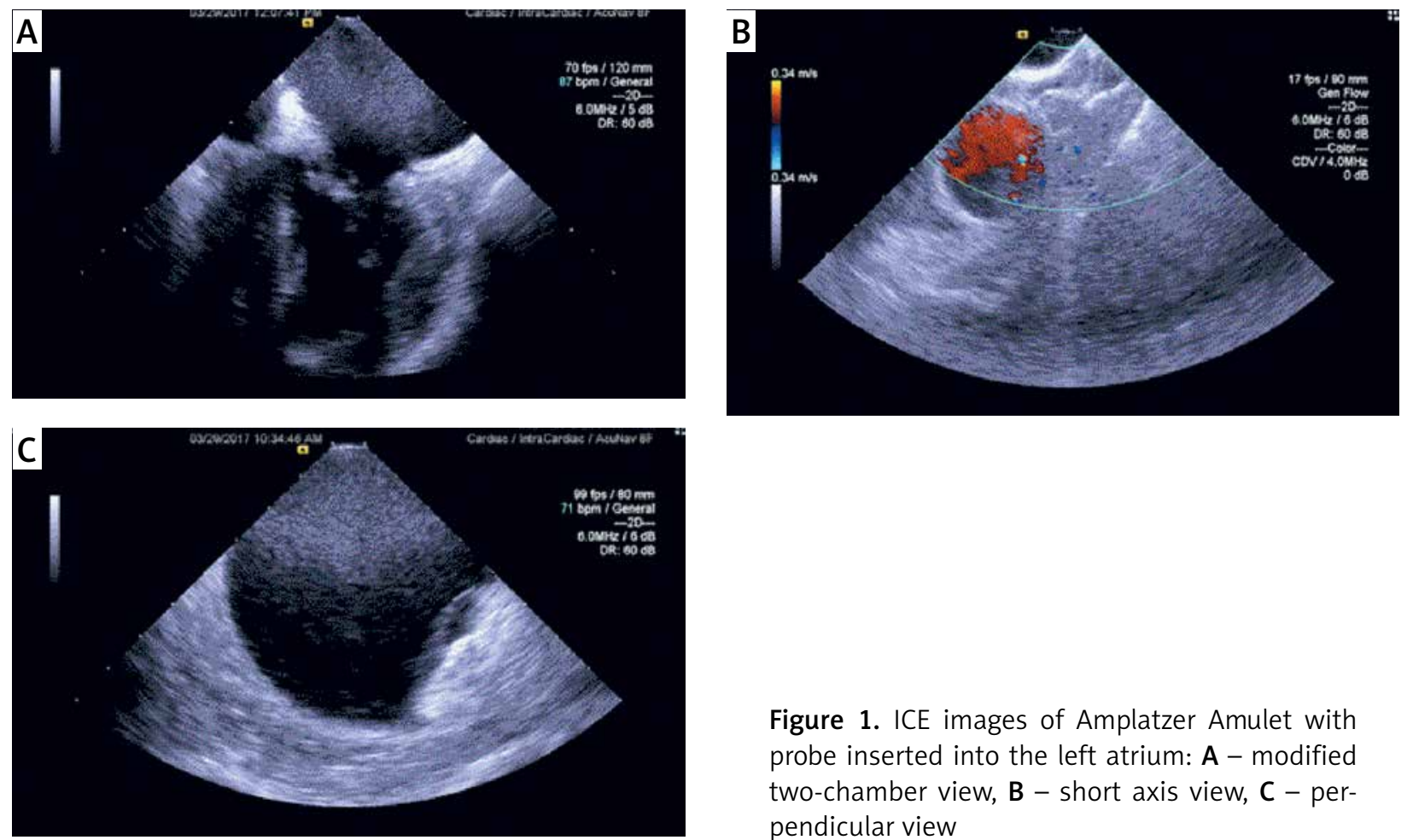

Figure 1. ICE images of Amplatzer Amulet with probe inserted into the left atrium: $\mathbf{A}$ - modified two-chamber view, B - short axis view, C - perpendicular view 


\section{Follow-up}

The follow-up was planned 30 days after implantation. The information regarding the outcome events (stroke, hemorrhage, late vascular complications) was gathered. All of the participating patients had a follow-up TOE to assess the result of implantation and to exclude thrombus in the left atrium before termination of the dual antiplatelet therapy.

\section{Statistical analysis}

Statistical analysis was performed using the Statistica ver. 12 (StatSoft Inc.) software. The quantitative data are presented as median and interquartile ranges, and the categorical data are reported as percentages. For the comparison the $\chi^{2}$ or Mann-Whitney $U$-test was used. Values of $p<0.05$ were considered statistically significant.

\section{Results}

The group in which LAAO was guided by TOE did not differ significantly from the ICE group regarding the demographic characteristics. The median age was respectively 73.00; IQR = 15.00 vs. $77.00 ; \mathrm{IQR}=7.00(p=0.69)$, and the percentage of males was $33.33 \%$ vs. $45.45 \%$ $(p=0.55)$.

$45.45 \%$ of patients in the ICE group and $66.66 \%$ in the TOE group had a paroxysmal atrial fibrillation $(p=0.31)$. The majority of the patients referred for LAAO had a history of major bleeding as the main indication for the procedure. Only 4 of 23 patients ( 2 in each group) had other indications for LAAO than serious bleeding. When comparing the risk of bleeding based on HASBLED score it was higher in the ICE group $(3.00 ; \mathrm{IQR}=1.00)$ than in the TOE group (2.00; $I Q R=0.50), p=0.01$. No significant differences between the groups were found with respect to the risk of stroke estimated based on the $\mathrm{CHA}_{2} \mathrm{DS}_{2} \mathrm{VASc}$ score. Mean $\mathrm{CHA}_{2} \mathrm{DS}_{2}$ VASC score was $5.00 ; \mathrm{IQR}=2.00$ in the ICE group and 5.00; IQR = 1.50 in the TOE group, $p=0.45$. Details regarding co-morbidities are shown in Table I.
The comparison of transthoracic echocardiography showed that both groups were similar according to dimensions and function of the left ventricle. LAAO guided by neither TOE nor ICE could be influenced by the morphology of the left atrial appendage. The details regarding left atrial appendage morphology, sizes and its relation to adjacent structures within the left atrium are given in Table II. The only significant difference was found for the area of the left atrial ostium, which was larger in the ICE group.

Time of the entire LAAO procedure ranged from 43 to $80 \mathrm{~min}$ in the TOE group with median procedure time 54 min, whereas in the ICE group the time from obtaining the first venous access to leaving the cathlab ranged from 28 to $67 \mathrm{~min}$ (median: $45 \mathrm{~min}$ ). The procedure time was significantly longer in the TOE group $(p=0.02)$. The time needed to puncture the interatrial septum starting from the first femoral access was insignificantly shorter in the ICE group $(p=0.09)$. The comparison of time that elapsed from gaining the first access to the femoral vein to withdrawal of the last sheath from the groin did not reveal statistically significant differences between the groups ( $p=0.97)$. The results are illustrated in Figure 2 .

During LAAO the proper position of the occluder was hardly ever achieved after the first deployment. It took place only in $2(16.67 \%)$ cases in the TOE group, and in none of the patients in the ICE group. In 2 cases (1 in the TOE group and 1 in the ICE group) it was necessary to change the occluder for another size. Finally successful occlusion of the left atrial appendage was achieved in all patients in TOE and ICE groups. The groups did not differ with regard to the size of occluder which was implanted (median: 28.00; $\mathrm{IQR}=3.00 \mathrm{~mm}$ vs. $25.00 ; \mathrm{IQR}=1.50 \mathrm{~mm} ; p=0.16$ ).

Crossing the intra-atrial septum required manipulation with the ICE probe and alignment of its position under fluoroscopy. It could explain the longer fluoroscopic time in the ICE group $(10.00 ; \mathrm{IQR}=8.00 \mathrm{~s})$ compared to the TOE group (7.00; IQR = 1.50s), but it was not statistically significant $(p=0.16)$. Also the lack of multi-plane

Table I. Characteristics of study population

\begin{tabular}{|c|c|c|c|}
\hline Parameter & ICE group & TOE group & $P$-value \\
\hline Coronary artery disease & $5(45.45 \%)$ & $7(58.33 \%)$ & 0.56 \\
\hline Diabetes mellitus & $3(27.27 \%)$ & $3(25.00 \%)$ & 0.96 \\
\hline History of ischemic stroke & $4(36.36 \%)$ & $3(25.00 \%)$ & 0.58 \\
\hline History of hemorrhagic stroke & $1(9.09 \%)$ & $0(0.00 \%)$ & 0.33 \\
\hline Heart failure & $2(20.0 \%)$ & $4(33.33 \%)$ & 0.52 \\
\hline Hypertension & $9(81.82 \%)$ & $11(91.67 \%)$ & 0.53 \\
\hline Uncontrolled hypertension & $2(18.18 \%)$ & $0(0.00 \%)$ & 0.14 \\
\hline Chronic kidney disease & $3(27.27 \%)$ & $1(8.33 \%)$ & 0.26 \\
\hline Fragility syndrome & $0(0.00 \%)$ & $1(8.33 \%)$ & 0.38 \\
\hline
\end{tabular}


Table II. Results of transthoracic and esophageal echocardiography performed prior to the procedure

\begin{tabular}{|c|c|c|c|}
\hline Parameter & TOE group & ICE group & $P$-value \\
\hline Minimal diameter of ostium $[\mathrm{mm}]^{\star}$ & $21.0(4.00)$ & $23.0(2.00)$ & 0.07 \\
\hline Maximal diameter of ostium $[\mathrm{mm}]^{*}$ & $27.5(5.00)$ & $31.0(7.00)$ & 0.08 \\
\hline Minimal diameter of landing zone $[\mathrm{mm}]^{*}$ & $17.0(3.00)$ & $17.0(3.00)$ & 0.59 \\
\hline Maximal diameter of landing zone $[\mathrm{mm}]^{\star}$ & $20.0(2.50)$ & $22.0(7.00)$ & 0.24 \\
\hline LAA ostium area $\left[\mathrm{cm}^{2}\right]^{*}$ & $4.7(1.16)$ & $5.5(1.30)$ & 0.04 \\
\hline LAA depth $[\mathrm{mm}]^{\star}$ & $21.5(7.50)$ & $24.0(10.0)$ & 0.62 \\
\hline \multicolumn{4}{|l|}{ Morphology of LAA: } \\
\hline Chicken wing & $5(41.67 \%)$ & $2(18.18 \%)$ & 0.21 \\
\hline Windsock & $6(50.00 \%)$ & $8(72.73 \%)$ & 0.26 \\
\hline Cauliflower & $1(8.33 \%)$ & $0(0.00 \%)$ & 0.33 \\
\hline Cactus & $0(0.00 \%)$ & $1(9.09 \%)$ & 0.29 \\
\hline \multicolumn{4}{|l|}{ LLR: } \\
\hline Sharp and tall & $11(91.67 \%)$ & $11(100 \%)$ & 0.31 \\
\hline \multicolumn{4}{|c|}{ Location of LAA ostium in relation to left pulmonary veins: } \\
\hline High & $2(16.67 \%)$ & $3(27.27 \%)$ & 0.56 \\
\hline Intermediate & $8(67.67 \%)$ & $5(45.45 \%)$ & 0.27 \\
\hline Low & $2(16.67 \%)$ & $3(27.27 \%)$ & 0.56 \\
\hline End diastolic dimension of left ventricle $[\mathrm{mm}]^{*}$ & $47.50(9.00)$ & $47.0(11.00)$ & 0.62 \\
\hline End systolic dimension of left ventricle $[\mathrm{mm}]^{*}$ & $35.0(4.00)$ & $32.0(12.00)$ & 0.44 \\
\hline Left ventricular ejection fraction [\%] ${ }^{\star}$ & $53.5(16.50)$ & $55.0(14.00)$ & 0.54 \\
\hline Left atrial diameter $[\mathrm{mm}]^{*}$ & $43.5(8.50)$ & $40.0(7.00)$ & 0.19 \\
\hline Left atrial area $[\mathrm{mm}]^{*}$ & $24.85(3.35)$ & $24.0(8.40)$ & 0.65 \\
\hline
\end{tabular}

*Median with interquartile range (IQR).

imaging with the ICE probe could result in more attempts to better visualize the left atrial appendage on fluoroscopy resulting in both prolonged fluoroscopic time and significantly higher contrast media volume used in the ICE group (30.00; IQR = $25.00 \mathrm{ml}$ vs. 20.00; IQR = $4.50 \mathrm{ml}$, $p=0.03$ ) (Figure 3).

Complications of the procedure in the studied population were very uncommon. No cardiac tamponade, pericardial effusion, device embolization, or periprocedural stroke was observed. Also very infrequent were vascular complications at the site of access. There was observed only 1 case of hematoma in the groin, which did not require additional treatment. No arterio-venous fistula or pseudoaneurysm was found after the procedure. Lack of complications allowed early mobilization and discharge from hospital. In the studied population the TOE group and ICE group did not differ regarding the time of hospitalization (3.00; IQR = 1.000 day vs. 3.00; IQR = 1.00 day; $p=0.71)$ or the time of immobilization $(6.00 ; \mathrm{IQR}=6.00 \mathrm{~h}$ vs. 8.0; IQR $=4.00 \mathrm{~h} ; p=0.74$ ).
After 1 month follow-up no stroke, bleeding or new vascular complications were reported. The follow-up analysis of TOE included the assessment of occluder position, the alignment of the device disk to the tissue of the appendage orifice, the presence of peridevice leak or thrombus on the surface of the occluder. We also categorized the effect of implantation as optimal if no left lateral ridge was left above the disk of the occluder or as suboptimal if part of the left lateral ridge was present above the disk. The detailed results of a follow-up TOE are shown in Table III. There were no statistically significant differences in the results of occluder implantation between TOE and ICE groups with respect to TOE follow-up assessments.

\section{Discussion}

According to recent studies, percutaneous obliteration of the left atrial appendage might help to reduce the risk of thromboembolism in non-valvular atrial fibrillation 
A

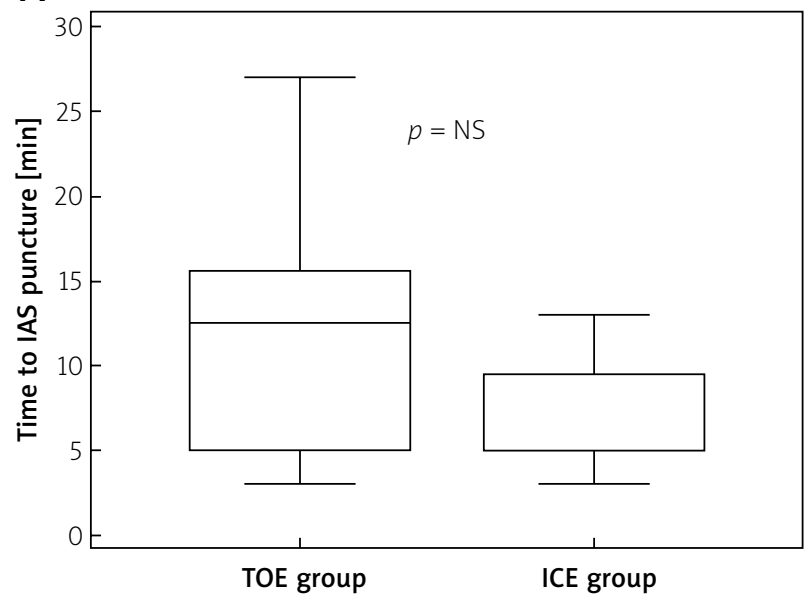

C

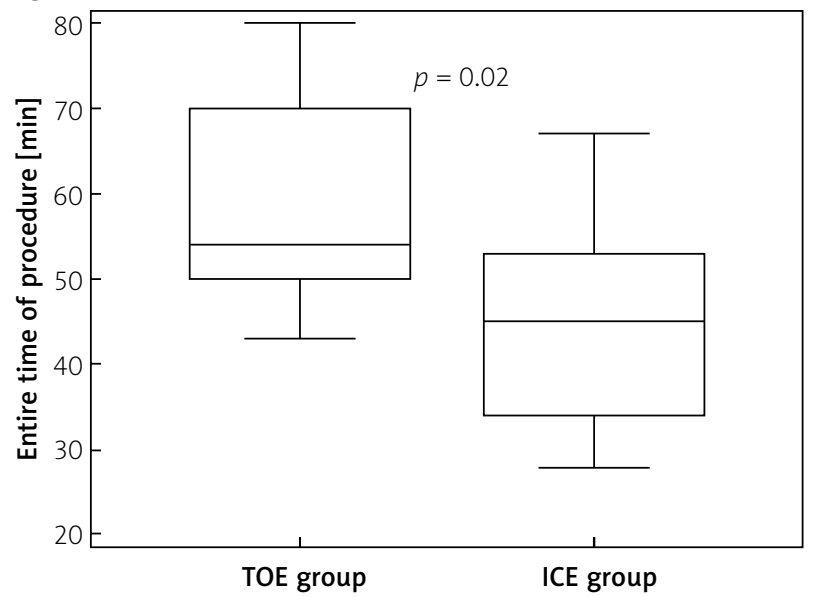

B

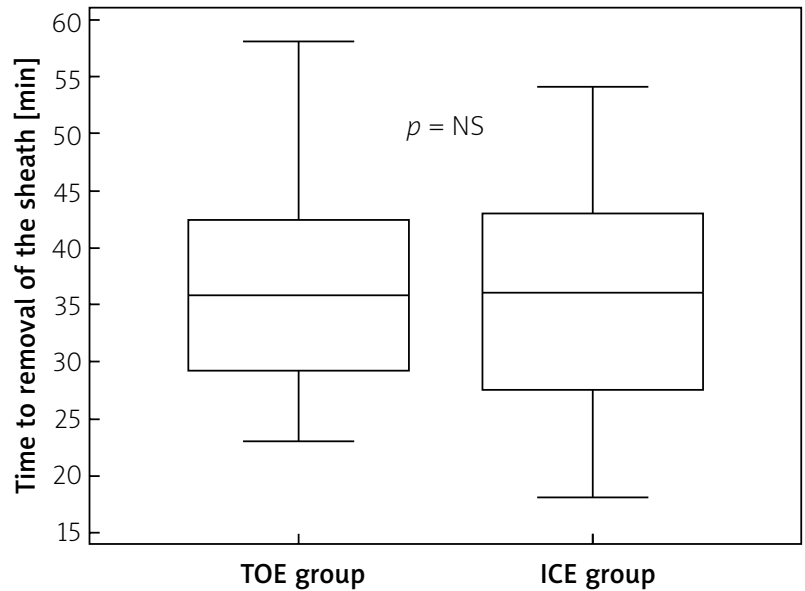

Figure 2. Time consumption at different stages of percutaneous left atrial appendage occlusion comparison of transesophageal and intracardiac echocardiographic guidance

TOE - transesophageal echocardiography, ICE - intracardiac echocardiography.

A

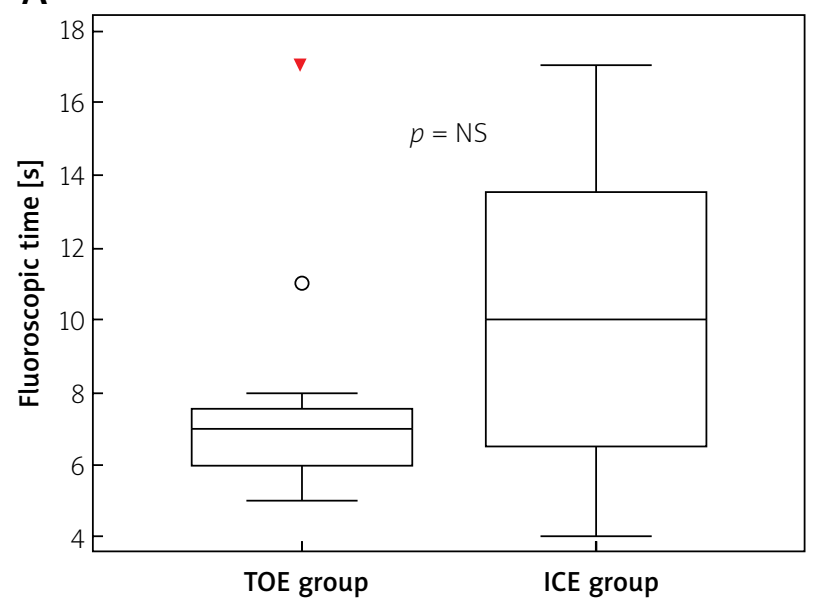

B

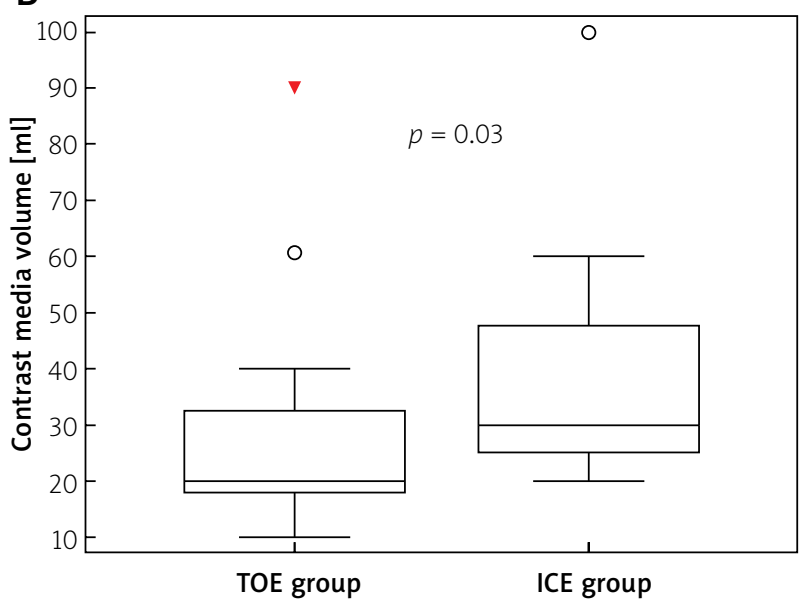

Figure 3. Fluoroscopic time and contrast media volume used for percutaneous left atrial appendage occlusion guided by transesophageal and intracardiac echocardiography

TOE - transesophageal echocardiography, ICE - intracardiac echocardiography. 
Table III. Results of follow-up transesophageal assessment

\begin{tabular}{lccc} 
Parameter & TOE group & ICE group & $P$-value \\
\hline Optimal position of occluder & $8(66.67 \%)$ & $6(54.54 \%)$ & 0.55 \\
\hline Occluder disk protruding to LAA & $0(0.00 \%)$ & $0(0.00 \%)$ & 1.00 \\
\hline Peridevice leak: & & & 1.00 \\
\hline Large & $0(0.00 \%)$ & $0(0.00 \%)$ & 0.59 \\
\hline Small & $2(16.67 \%)$ & $1(9.09 \%)$ & 0.59
\end{tabular}

$[7,8]$. Promising results have been obtained with occluders offered by various manufacturers and nowadays it may be considered by the guidelines as a valuable alternative for patients with a high risk of stroke and contraindications for long-term oral anticoagulation [9-12].

Independently from the type of occluder used, the procedure of LAAO requires an adequate imaging modality for both procedure planning and guiding. Often the usage of fluoroscopy alone to guide LAAO is insufficient, particularly in cases of complex LAA. Thus TOE has become a gold standard technique for management of LAAO, but also this modality has some disadvantages [13]. TOE necessitates the use of general anesthesia or deep sedation, and an experienced TOE echocardiographer and anesthetic team. Thus newer imaging modalities, such as ICE, appear to be a good alternative [14].

Usefulness of ICE was confirmed in periprocedural guiding of such procedures as left heart ablation, closure of interatrial communications, the placement of percutaneous left ventricular assist device cannulas, the performance of percutaneous balloon mitral valvuloplasty, transcatheter aortic valve implantation, and some others [15-17]. Recently there has also been growing interest in application of ICE in guiding of LAAO procedures. It has also been demonstrated that ICE may serve as an alternative to the TOE imaging modality for detecting left atrial appendage thrombus prior to procedures within the left atrium [18, 19].

Compared to TOE, ICE can visualize the left atrial appendage more clearly as a result of higher image resolution. The ICE probe can remain in place within the right or left heart safely for the entire procedure with excellent patient tolerance. Modern ICE probes provide easy applicability, and real-time structural and hemodynamic information, which allow not only the assessment of anatomy but also detection of residual flow after the deployment of the left atrial appendage occluder.

There are a few studies showing that ICE guiding for percutaneous closure of interatrial communications reduces the fluoroscopy time, interventional procedure time, and catheterization laboratory time compared with TOE $[20,21]$. In the present study a precise analysis of procedure timing was performed. Similarly to mentioned pediatric procedures in our series the entire time of the procedure in the ICE group, counted starting at the venous puncture and ending when the patient left the cathlab, was significantly lower compared to the TOE group. Data regarding the procedure time during LAAO guided by ICE were published by Frangieh et al. [22]. In this study the reported time from femoral venous puncture to transseptal puncture and to closure was longer in the ICE group. In this study, of the TOE-guided LAAO, 17 were combined with TAVI, and 6 procedures in the ICE group were combined with percutaneous coronary interventions. Thus the results are not comparable to our series. Similarly, Berti et al. showed that TEE implied lower procedural and fluoroscopy time when compared with ICE [23]. In our study the time from venous puncture to transseptal puncture was not significantly different between groups, and the reduction of the entire procedure time in the ICE group was mainly achieved by the lack of need for the patient's awareness recovery from anesthesia in the ICE group. More consistent data were analyzed recently by Korsholm et al., who similarly showed that LAAO guided by ICE was associated with shorter duration of the procedure [24]. However, different time intervals of the LAAO procedure were used; thus no direct comparison with our results can be performed.

Similarly to TOE, ICE also allows direct monitoring of acute procedure-related complications (e.g., thrombus formation, pericardial effusion). On the other hand, ICE carries some potential disadvantages. The most important possible complications are those associated with right heart catheterization such as pulmonary embolism, pericardial tamponade, but also bleeding from the puncture site, and vascular complications. Moreover, the mono-plane images may limit the usefulness of ICE in left atrial appendage occluder sizing [4].

Matsuo et al. demonstrated that ICE is an effective and safe method of guiding the LAAO procedure. In a series of 27 patients who underwent LAAO, the Watchman device was successfully implanted in all cases and no major complications were reported [25]. Minor complications, which occurred in 4 (14.8\%) patients, were mainly hematoma of the groin (3 cases, 11.1\%). However, implantation of the Amplatzer Amulet requires a different 
assessment during the procedure. Unlike the Watchman device, which can be implanted deep in the appendage, the Amplatzer Amulet disk should cover the left atrial appendage ostium. Thus the mono-plane imaging by ICE, and restricted ability to rotate the probe, may limit the usefulness of ICE for guiding the Amplatzer Amulet implantation. Masson et al. published data on the safety and efficacy of a prototype of the Amplatzer Amulet - the Amplatzer Cardiac Plug, which was implanted under the guidance by ICE from the left atrium [26]. Procedural success was achieved in 36 of 37 patients (97\%). The authors reported 3 major complications (tamponade, myocardial stroke and major bleeding), which is similar to that of published registries for the same devices but under TOE guidance $[27,28]$. The impact of the learning curve was also observed in the analyzed series. Frangieh et al. [22] compared percutaneous occlusion of the left atrium with the Watchman device that was deployed either under ICE (32 patients, $42 \%$ ) or under TOE (44 patients, 58\%). The device implantation success rate was $100 \%$ in both groups. Total contrast media injected during LAAO as well as fluoroscopy time were comparable between both groups. In contrast, in the present study the fluoroscopic time was insignificantly longer in the ICE group, which may be explained by the need of fluoroscopic assessment of ICE probe position. Also the amount contrast medium used during LAAO was larger in the ICE group, and the difference reached statistical significance. It can be explained by lack of experience with ICE, which resulted in an increase of views performed under fluoroscopy to assess the left atrial appendage.

Berti et al. [29] showed that ICE was able to perform the tasks typically provided by TEE during implantation of the Amplatzer Cardiac Plug device for left atrial appendage occlusion, such as assessment of its dimension for device sizing, guidance of transseptal puncture, verification of the delivery sheath position, confirmation of location and stability of the device. But contrary to previous studies the procedural success was lower, and it was achieved in 113 of 121 patients (93.4\%). The procedural success rate was lower compared to the present data in the ICE group, but a different approach for ICE was used. Instead of insertion of the ICE probe to the left atrium the ICE probe was positioned in the right atrium or in the coronary sinus. Such a position of the probe may result in poorer quality of images; thus the post-deployment assessment was more difficult.

\section{Study limitations}

The main study limitation is the small number of patients included in the study. The results might also be influenced by the fact that the operators had long experience in LAAO procedures as well as in TOE guidance of LAAO, whereas the experience with ICE was very limited.

\section{Conclusions}

LAAO using the Amplatzer Amulet may be successfully and safely guided by ICE. Despite the lack of anesthesia or sedation, ICE offered a shorter procedure time compared to TOE guidance. Suboptimal implantation of the occluder, as well as presence of leak or thrombus, was seen with the same incidence in both groups.

\section{Acknowledgments}

The study was supported by a grant from the Silesian Medical University in Katowice (grant number KNW-1$119 / \mathrm{N} / 7 / K)$.

\section{Conflict of interest}

W. Streb, K. Mitręga and Z. Kalarus are proctors of Abott Medical Sp. z o.o.

The other authors declare no conflict of interest.

\section{References}

1. Marchandise S, Kefer J, le Polain de Waroux JB, Jean-Louis Vanoverschelde CS. Role of cardiac imaging for catheter-based left atrial appendage closure. J Atr Fibrillation 2013; 6: 702.

2. Manning WJ, Weintraub RM, Waksmonski CA, et al. Accuracy of transesophageal echocardiography for identifying left atrial thrombi: a prospective, intraoperative study. Ann Intern Med 1995; 123: 817-22.

3. Klein AL, Murray RD, Grimm RA. Role of transesophageal echocardiography-guided cardioversion of patients with atrial fibrillation. J Am Coll Cardiol 2001; 37: 691-704.

4. Chow DHF, Bieliauskas G, Sawaya FJ, et al. A comparative study of different imaging modalities for successful percutaneous left atrial appendage closure. Open Heart 2017; 4: e000627.

5. Wazni OM, Tsao HM, Chen SA, et al. Cardiovascular imaging in the management of atrial fibrillation. J Am Coll Cardiol 2006; 48: 2077-84.

6. Gafoor S, Schulz P, Heuer L, et al. Use of echonavigator, a novel echocardiography-fluoroscopy overlay system, for transseptal puncture and left atrial appendage occlusion. J Interv Cardiol 2015; 28: 215-7.

7. Holmes DR, Reddy VY, Turi ZG, et al. Percutaneous closure of the left atrial appendage versus warfarin therapy for prevention of stroke in patients with atrial fibrillation: a randomised non-inferiority trial. Lancet 2009; 374: 534-42.

8. Reddy VY, Holmes D, Doshi SK, et al. Safety of percutaneous left atrial appendage closure: results from the Watchman Left Atrial Appendage System for Embolic Protection in Patients with AF (PROTECT AF) clinical trial and the Continued Access Registry. Circulation 2011; 123: 417-24.

9. Tzikas A, Shakir S, Gafoor S, et al. Left atrial appendage occlusion for stroke prevention in atrial fibrillation: multicentre experience with the AMPLATZER Cardiac Plug. Eurointervention 2016; 11: 1170-9.

10. Reddy V, Price $M$, Valderrabano $M$, et al. Long-term event rates for left atrial appendage closure with watchman: Protect AF 5 year and prevail 3 year follow-up. J Am Coll Cardiol 2016; 68: B11.

11. Meier B, Blaauw Y, Khattab AA, et al. EHRA/EAPCI expert consensus statement on catheter-based left atrial appendage occlusion. Europace 2014; 16: 1397-416. 
12. Kirchhof P, Benussi S, Kotecha D, et al. 2016 ESC Guidelines for the management of atrial fibrillation developed in collaboration with EACTS. Rev Esp Cardiol 2017; 70: 50.

13. Chue CD, de Giovanni J, Steeds RP. The role of echocardiography in percutaneous left atrial appendage occlusion. Eur J Echocardiogr 2011; 12: i3-10.

14. Clemente A, Avogliero F, Berti S, et al. Multimodality imaging in preoperative assessment of left atrial appendage transcatheter occlusion with the Amplatzer Cardiac Plug. Eur Heart J Cardiovasc Imaging 2015; 16: 1276-87.

15. Ren JF, Marchlinski FE, Callans DJ, Herrmann HC. Clinical use of AcuNav diagnostic ultrasound catheter imaging during left heart radiofrequency ablation and transcatheter closure procedures. J Am Soc Echocardiogr 2002; 15: 1301-8.

16. Silvestry FE, Kerber RE, Brook MM, et al. Echocardiography-guided interventions. J Am Soc Echocardiogr 2009; 22: 213-31.

17. Bartel T, Müller S, Biviano A, Hahn RT. Why is intracardiac echocardiography helpful? Benefits, costs, and how to learn. Eur Heart J 2014; 35: 69-76.

18. Ikegami Y, Tanimoto K, Inagawa K, et al. Identification of left atrial appendage thrombi in patients with persistent and longstanding persistent atrial fibrillation using intra-cardiac echocardiography and cardiac computed tomography. Circ J 2017; 82: 46-52.

19. Baran J, Zaborska B, Piotrowski R, et al. Intracardiac echocardiography for verification for left atrial appendage thrombus presence detected by transesophageal echocardiography: the ActionICE II study. Clin Cardiol 2017; 40: 450-4.

20. Bartel T, Konorza T, Arjumand J, et al. Intracardiac echocardiography is superior to conventional monitoring for guiding device closure of interatrial communications. Circulation 2003; 107: 795-7.

21. Boccalandro F, Baptista E, Muench A, et al. Comparison of intracardiac guidance for percutaneous transcatheter closure of atrial septal defect. Am J Cardiol 2004; 93: 437-40.

22. Frangieh AH, Alibegovic J, Templin C, et al. Intracardiac versus transesophageal echocardiography for left atrial appendage occlusion with watchman. Catheter Cardiovasc Interv 2017; 90: 331-8.

23. Berti S, Pastormerlo LE, Santoro G, et al. Intracardiac versustransesophageal echocardiographic guidence for left atrial appendage occlusion. The LAAO Italian Multicenter Registry. JACC Cardiovasc Interv 2018; 11: 1086-92.

24. Korsholm K, Jensen JM, Nielsen-Kudsk JE. Intracardiac echocardiography from the left atrium for procedural guidance of transcatheter left atrial appendage occlusion. JACC Cardiovasc Interv 2017; 10: 2198-206.

25. Matsuo Y, Neuzil P, Petru J, et al. Left atrial appendage closure under intracardiac echocardiographic guidance: feasibility and comparison with transesophageal echocardiography. J Am Heart Assoc 2016; 5: e003695.

26. Masson J, Kouz R, Riahi M, et al. Transcatheter left atrial appendage closure using intracardiac echocardiographic guidance from the left atrium. Can J Cardiol 2015; 31: 1497.e7-14.

27. Park J, Bethencourt A, Sievert $\mathrm{H}$, et al. Left atrial appendage closure with amplatzer cardiac plug in atrial fibrillation: initial European experience. Catheter Cardiovasc Interv 2011; 77: 700-6.

28. Lopez-Mingues JR, Eldoayen-gragera J, Gonzalez-Fernandez R, et al. Immediate and one-year results in 35 consecutive patients after closure of left atrial appendage with the Amplatzer Cardiac Plug. Rev Esp Cardiol 2013; 66: 90-7.
29. Berti S, Paradossi U, Meucci F, et al. Periprocedural intracardiac echocardiography for left atrial appendage closure: a dual-center experience. JACC Cardiovasc Interv 2014; 7: 1036-44. 